\title{
Brain MR Findings in Patients with Systemic Lupus Erythematosus with and without Antiphospholipid Antibody Syndrome
}

\author{
Y. Kaichi, S. Kakeda, J. Moriya, N. Ohnari, K. Saito, Y. Tanaka, F. Tatsugami, S. Date, K. Awai, and Y. Korogi
}

\begin{abstract}
BACKGROUND AND PURPOSE: Antiphospholipid syndrome may affect the incidence and pathogenesis of cerebrovascular diseases in patients with systemic lupus erythematosus. We compared the spectrum of MR findings in patients with systemic lupus erythematosus with and without antiphospholipid syndrome.
\end{abstract}

MATERIALS AND METHODS: We identified 256 patients with systemic lupus erythematosus (45 with, 211 without antiphospholipid syndrome) who underwent MR studies; in 145 (57\%), we detected abnormalities. These were categorized as large territorial, lacunar, localized cortical, and borderzone infarctions and as microembolisms, basal ganglia lesions, callosal lesions, hemorrhages, and white matter hyperintensity on T2-weighted and/or FLAIR images, and as stenotic arterial lesions on MR angiograms. Logistic regression analysis was performed to compare the MR findings in patients with systemic lupus erythematosus with and without antiphospholipid syndrome, with patient age and antiphospholipid syndrome as the covariates.

RESULTS: Abnormal MR findings were more common in patients with systemic lupus erythematosus with antiphospholipid syndrome (73\% versus 53\%). Large territorial $(P=.01)$, lacunar $(P=.01)$, localized cortical $(P<.01)$, borderzone infarcts $(P<.01)$, basal ganglia lesions $(P=.03)$, stenotic arterial lesions $(P=.04)$, and the rate of positive findings on MR imaging $(P=.01)$ were significantly associated with antiphospholipid syndrome. Irrespective of age, significantly more patients with antiphospholipid syndrome manifested lacunar infarcts in the deep white matter $(P<.01)$, localized cortical infarcts in the territory of the MCA $(P<.01)$, bilateral borderzone infarcts $(P<.01)$, and anterior basal ganglia lesions $(P=.01)$.

CONCLUSIONS: Abnormal MR findings were more common in patients with systemic lupus erythematosus with than in those without antiphospholipid syndrome. Large territorial infarctions, lacunar infarctions in the deep white matter, localized cortical infarctions in the MCA territory, bilateral borderzone infarctions, anterior basal ganglia lesions, and stenotic arterial lesions are common MR findings in patients with systemic lupus erythematosus with antiphospholipid syndrome.

ABBREVIATIONS: aPL = antiphospholipid antibodies; APS = antiphospholipid syndrome; GRE = gradient echo; SLE = systemic lupus erythematosus; WMH = white matter hyperintensity

S ystemic lupus erythematosus (SLE) is an autoimmune disease that frequently manifests with involvement of the central nervous system. ${ }^{1,2}$ A previous autopsy study of neuropsychiatric SLE revealed various types of brain lesions including global ischemic changes, parenchymal edema, microhemorrhages, glial

Received September 12, 2012; accepted after revision April 5, 2013.

From the Department of Radiology (Y. Kaichi, S.K., J.M., N.O., Y. Korogi) and First Department of Internal Medicine (K.S., Y.T.), University of Occupational and Environmental Health, School of Medicine, Kitakyushu, Japan; and Department of Diagnostic Radiology (Y. Kaichi, F.T., S.D., K.A.), Graduate School of Biomedical Sciences, Hiroshima University, Hiroshima, Japan.

Please address correspondence to Shingo Kakeda, MD, PhD, Department of Radiology, University of Occupational and Environmental Health, 1-one Iseigaoka, Yahatanishi-ku, Kitakyushu 807-8555, Japan; e-mail: kaichi@hiroshima-u.ac.jp

三 Indicates article with supplemental on-line tables.

http://dx.doi.org/10.3174/ajnr.A3645 hyperplasia, diffuse neuronal/axonal loss, resolved infarction, microthromboemboli, blood vessel remodeling, acute infarction, acute macrohemorrhages, and resolved intracranial hemorrhages. ${ }^{3}$ A wide spectrum of MR findings in patients with SLE has also been reported. ${ }^{3}$ SLE is a heterogeneous disease characterized by multisystem autoimmunity, leading to an array of clinical presentations. In addition, there is also a small subset of patients with SLE who show persistently negative antinuclear antibody tests despite having the typical clinical features of SLE. These variabilities can add to the difficulty of timely diagnosis and intervention. ${ }^{4}$ Understanding the wide spectrum of brain pathologic conditions in patients with SLE may help to render an appropriate diagnosis.

Antiphospholipid syndrome (APS) is characterized by antiphospholipid antibodies (aPL) and specific thromboembolic phenomena, including deep venous thrombosis and spontaneous 
abortions. ${ }^{5}$ However, both events are relatively common in the general population and in subjects with autoimmune diseases. Moreover, in patients with thrombosis or spontaneous abortion in whom APS is strongly suspected, conventional aPL are repeatedly negative. ${ }^{6}$ This condition has been called "seronegative APS."7 Therefore, the correct identification of patients with APS can be a complex task. The diagnosis of APS affects treatment options; an antiplatelet and/or anticoagulation therapy is recommended for neuropsychiatric SLE related to aPL, especially for thrombotic cerebrovascular disease. ${ }^{8}$ Therefore, it is important to investigate MR findings in patients with SLE with APS and those without APS. The association of aPL/APS with neurologic involvement has been established, ${ }^{9,10}$ but there has been limited reporting of differences in MR findings in patients with SLE with APS and those without APS. ${ }^{11}$ That study concluded that infarc-

\section{MRI findings in patients with SLE}

\begin{tabular}{|c|c|c|c|c|}
\hline \multirow[b]{2}{*}{ MRI findings } & \multicolumn{2}{|c|}{$\begin{array}{l}\text { Patients With } \\
\text { SLE, No. (\%) }\end{array}$} & \multicolumn{2}{|c|}{$\begin{array}{c}\text { Occlusions or Stenotic } \\
\text { Lesions of Arteries, } \\
\text { No. (\%) }\end{array}$} \\
\hline & $(n=256)$ & $(\%)$ & & $(\%)$ \\
\hline Infarction/infarction-like lesions & 69 & (27) & 8 & (12) \\
\hline Large territorial infarctions & 23 & (9) & 6 & (26) \\
\hline Lacunar infarctions & 23 & (9) & 2 & (9) \\
\hline Localized cortical infarctions & 19 & (7) & 2 & (11) \\
\hline Borderzone infarctions & 11 & (4) & 2 & (18) \\
\hline Basal ganglia lesions & 11 & (4) & 0 & 0 \\
\hline Callosal lesions & 3 & (1) & 1 & (33) \\
\hline Acute microemboli & 4 & (2) & 0 & 0 \\
\hline Other lesions & 118 & (46) & & \\
\hline Hemorrhages & 6 & $(2)$ & & \\
\hline WMH & 108 & $(42)$ & & \\
\hline Stenotic arterial lesions & 9 & (4) & & \\
\hline Abnormal findings & 145 & (57) & & \\
\hline
\end{tabular}
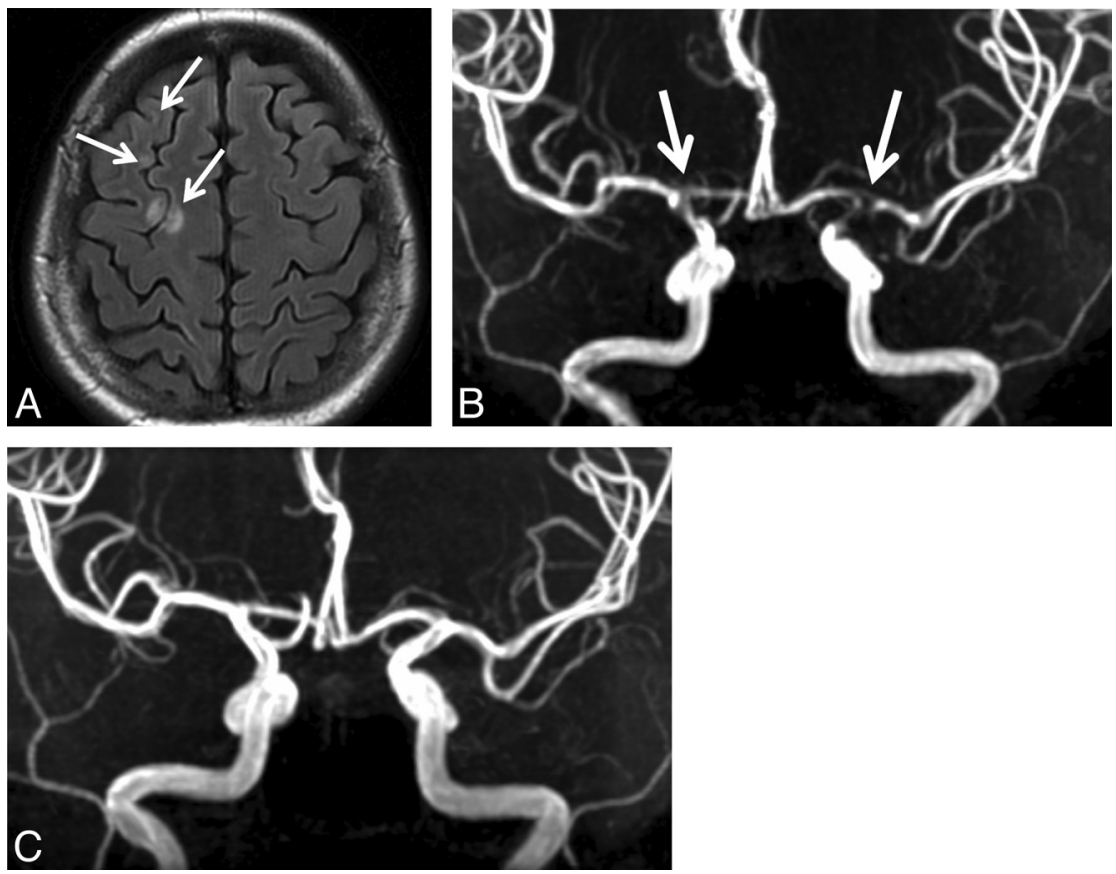

FIG 1. A 34-year-old woman with SLE with APS. A, Axial FLAIR image shows right borderzone infarction (arrows). B, MR imaging study performed 3 days after symptom onset shows evidence of marked narrowing of the bilateral internal carotid artery and the M1 segment of the bilateral MCA (arrows). C, Follow-up MRA study performed 60 days after symptom onset shows recovery of the arterial stenosis. tions and infarcts with white matter hyperintensity (WMH) were more common in patients with SLE with APS, but the study population was relatively small and the specific nature of the MR findings was not described in detail. We characterize the spectrum of MR findings in a large series of patients with SLE and compare our findings in patients with SLE with APS and those without APS.

\section{MATERIALS AND METHODS}

\section{Patients}

This retrospective study was approved by our institutional review board; informed consent was waived. We reviewed the data base of patient charts entered between May 2004 and June 2011 and selected 261 patients diagnosed with SLE on the basis of American Rheumatism Association criteria for the classification of SLE. ${ }^{12}$ At our institution, a screening brain MR imaging has been routinely performed for the assessment of patients with SLE.

Exclusion criteria included unsatisfactory images because of artifacts and a history of other neurologic disease. Thus, on the basis of these exclusion criteria, we eliminated 2 patients whose image quality was inadequate and 3 because they had a brain tumor, osmotic myelinolysis, or multiple sclerosis. Consequently, 256 patients with SLE who underwent brain MR study were included. Of these, $211(82.4 \%)$ did not and 45 (17.6\%) did have APS diagnosed according to Sapporo criteria. ${ }^{10,13}$

We reviewed patient demographic data for vascular risk factors (diabetes mellitus, defined as a random glucose level $>11.1$ $\mathrm{mmol} / \mathrm{L}$, a fasting blood glucose level $>7.0 \mathrm{mmol} / \mathrm{L}, \mathrm{HbAlc}$ $>6.5 \%$, or current use of antidiabetic drugs), hypertension (blood pressure $>140 / 90 \mathrm{~mm} \mathrm{Hg}$, or current treatment with antihypertensive drugs), past and current smoking, dyslipidemia (LDL cholesterol $>3.64 \mathrm{mmol} / \mathrm{L}$, HDL cholesterol $<0.91$ $\mathrm{mmol} / \mathrm{L}$, triglyceride $>1.7 \mathrm{mmol} / \mathrm{L}$, or receiving treatment), obesity (body mass index $>26 \mathrm{~kg} / \mathrm{m}^{2}$ ), duration of SLE (interval between diagnosis and brain MR study), and previous treatments (corticosteroids and immunomodulatory drugs).

\section{MR Imaging}

All studies were performed on a $1.5 \mathrm{~T}$ or 3T MR system (Signa Excite, GE Healthcare, Milwaukee, Wisconsin) by use of a dedicated 8-channel phased-array coil (USA Instruments, Aurora, Ohio). The imaging parameters were 4000/85/1/ 13.4/1 minute, 50 seconds (repetition time msec/echo time msec/NEX/echo spacing/imaging time), with an echotrain length of 14 at $1.5 \mathrm{~T}$, and $4500 / 85 /$ $1 / 10.9 / 2$ minutes, 10 seconds, with an echo-train length of 16 at $3 \mathrm{~T}$ for $\mathrm{T} 2$ weighted FSE imaging. They were $8000 /$ 115/2000/2/8.0/2 minutes, 40 seconds (repetition time msec/echo time $\mathrm{msec} /$ 
inversion time/NEX/echo spacing/imaging time), with an echotrain length of 30 at $1.5 \mathrm{~T}$, and 12,000/140/2600/2/9.1/3 minutes, 20 seconds, with an echo-train length of 30 at $3 \mathrm{~T}$ for FLAIR imaging. At both field strengths, T2-weighted and FLAIR images were acquired at a section thickness of $5 \mathrm{~mm}$, an intersection gap of $2.5 \mathrm{~mm}$, an FOV of $22 \mathrm{~cm}$, and a matrix of $256 \times 192$. In addition, all patients underwent our standard brain MR imaging protocol including T1WI, DWI, and 3D TOF intracranial MRA. The following imaging parameters at $3 \mathrm{~T}$ were used: 2500/10/90/ $320 \times 224 / 22 \times 22 / 1$ minute, 30 seconds (TR msec/TE msec/flip angle/section thickness/matrix/FOV/imaging time), for T1WI and 6000/minimum/not available/5/128 $\times 256 / 22 \times 22 / 30 \mathrm{sec}-$ onds for DWI. The imaging parameters for $3 \mathrm{D}$ TOF MRA were 30 $\mathrm{msec} / 6.3 \mathrm{msec} / 20^{\circ} / 1 \mathrm{~mm} / 18 \mathrm{~cm} / 256 \times 256 / 4$ minutes, 32 seconds (repetition time msec/echo time msec/flip angle/section thickness/FOV/matrix/imaging time), at $1.5 \mathrm{~T}$; they were $30 \mathrm{msec} / 3.3$ $\mathrm{msec} / 20^{\circ} / 1 \mathrm{~mm} / 18 \mathrm{~cm} / 384 \times 224 / 4$ minutes, 37 seconds, at $3 \mathrm{~T}$. In 29 of 256 patients with SLE (11\%), gradient echo (GRE) T2WI scans were obtained on $1.5 \mathrm{~T}$ or $3 \mathrm{~T}$ systems.

\section{MR Imaging Analysis}

Two neuroradiologists (S.K. and Y. Kaichi) reviewed the MR images and categorized their findings as normal (grade 1), as infarction or infarction-like lesions (large territorial, lacunar, localized cortical, or bilateral or unilateral borderzone infarctions; acute multiple or single micro-embolisms; basal ganglia; or callosal lesions; grade 2), and as other lesions (WMH, macro- or microhemorrhages, stenotic arterial lesions on MRA; grade 3). We found various kinds of destructive brain lesions in this study, for some of which we could not identify the specific MR findings suggesting cerebral infarctions according to the previous observations. Therefore, we defined them as infarction-like lesions.

Large territorial infarction ${ }^{14}$ was defined as an infarct that involved all or a large part of the territory of the anterior cerebral artery, MCA, or posterior cerebral artery; the cerebellum; or the brain stem in a characteristic pattern of a large vessel occlusion. The readers defined acute multiple or single microemboli as small hyperintense foci $<3 \mathrm{~mm}$ in the cortex and/or subcortical white matter on DWI. Localized cortical infarction ${ }^{15}$ was recorded when there were single or multifocal ischemic lesions in the cortex. Therefore, this category included chronic small cortical infarcts suggestive of microembolism and acute cortical lesions $>3$ mm. ${ }^{16}$

Borderzone infarction was defined according to the templates of Bogousslavsky and Regli ${ }^{17}$ and the atlas of Damasio. ${ }^{18}$ Lacunar infarction, defined as a CSF-filled cavity with increased peripheral signal on FLAIR $<15 \mathrm{~mm}$ in diameter, ${ }^{19}$ was subclassified into basal ganglia, deep white matter, or brain stem infarction. Lesions larger than lacunar infarcts were recorded as basal ganglia lesions; their location was divided into anterior, middle, and posterior. Callosal lesions were defined as abnormally hyperintense areas in any part of the corpus callosum on DWI, T2WI, or FLAIR scans. $\mathrm{WMH}$ was identified as ill-defined hyperintensity $\geq 5 \mathrm{~mm}$ on both T2-weighted FSE and FLAIR images, and WHM was rated according to the scale of Fazekas et al, ${ }^{20}$ in which grade $1=$ punctate foci, grade $2=$ incipient confluence of foci, and grade $3=$ large confluent areas. In the present study, we made a distinction between WMH and perivascular space on the basis of the MR findings in a previous report ${ }^{21}$; the perivascular space was visualized as hyperintense regions on T2WI and as hypointense regions on T1WI and formed punctuate or tubular structures of usually $<3 \mathrm{~mm}$ in diameter, and usually located in conformity with the path of perforating brain vessels, which run perpendicular to the brain surface. Micro- and macrohemorrhages were defined as hemorrhages $\leq 5 \mathrm{~mm}$ and $>5 \mathrm{~mm}$ in diameter on the basis of T2WI findings, because only 29 of the 256 patients (11\%) underwent GRE T2WI. ${ }^{22}$ Stenotic arterial lesions were recorded when there was either complete occlusion or stenosis of $>50 \%$ on MRA images. Because of the limited FOV and spatial resolution of MRA images, the assessed intracranial vessels included segments of the internal carotid artery, the middle cerebral artery (M1 [the sphenoidal segment], M2 [the insular segment], M3 [the opercular segment]), and the anterior cerebral artery (A1 [the segment from the origin of the anterior cerebral artery to the anterior communicating artery] and A2 [the infracallosal segment]).

\section{Statistical Analysis}

We compared the differences in the baseline characteristics of patients with SLE with and without APS by use of logistic regression analysis. To compare MR findings in patients with SLE with and without APS, logistic regression was also applied to correct for confounding age and APS, resulting in an adjusted odds ratio. Differences of $P<.05$ were considered statistically significant.

\section{RESULTS}

Whereas the demographic and clinical characteristics were not significantly different between patients with and without APS, the median disease duration was longer in those with APS $(P=.05)$ (On-line Table 1).

Our MR findings on all patients with SLE are summarized in the Table. The most common finding was WMH. Infarcts and infarct-like lesions were seen in 69 of the 256 patients (27\%); only 8 of the $69(12 \%)$ harbored stenotic lesions on major intracranial arteries. On MRA images, the relevant artery manifested stenotic lesions in $6(26 \%)$ of 23 patients with large territorial, 2 (9\%) of 23 with lacunar, and $2(18 \%)$ of 11 with borderzone infarcts. In 1 patient with borderzone infarction, the initial MRA scan showed marked narrowing of the bilateral internal carotid artery and the M1 segment of the bilateral MCA; follow-up MRA revealed resolution of the arterial stenoses. In this patient, we considered vasculitis or arterial vasospasm a likely diagnosis (Fig 1). No stenotic lesion on the relevant artery was seen in patients with basal ganglia lesions and acute micro-embolism in the cortex and/or subcortical white matter.

On-line Table 2 is a comparison of the MR imaging findings in patients with SLE with and without APS. More patients with than without APS demonstrated abnormal findings (73\% versus 53\%). The incidence of large territorial, lacunar, localized cortical, and borderzone infarcts; acute microemboli; basal ganglia lesions; callosal lesions; and stenotic arterial lesions was higher in patients with APS than in those without APS.

We performed logistic regression analysis to compare MR findings in patients with SLE with and without APS, with patient age and APS as the covariates. Large territorial, localized cortical 

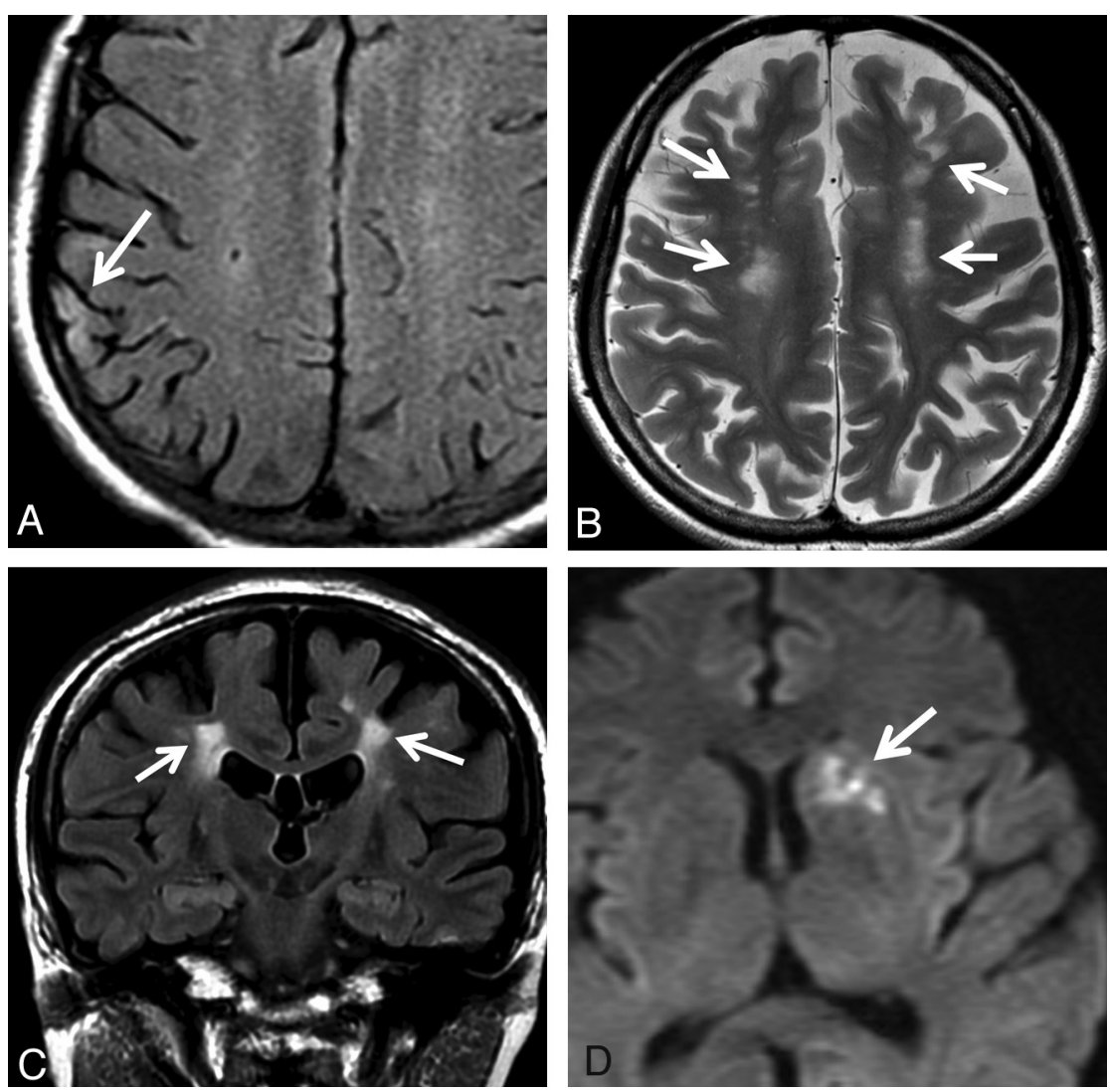

FIG 2. A, Sixteen-year-old boy with SLE with APS. Axial FLAIR image shows localized cortical infarction (arrow). B and C, Thirty-nine-year-old woman with SLE with APS. Axial T2WI (B) and coronal FLAIR image (C) show bilateral borderzone infarction (arrows). MRA revealed no abnormality (image not shown). D, Forty-five-year-old woman with SLE with APS. Axial DWI image shows a lesion in the left anterior basal ganglia (arrow).

(Fig 2A), and borderzone infarctions (Fig 2B, -C); basal ganglia lesions (Fig 2D); lacunar infarcts; stenotic arterial lesions; and the total number of patients with abnormal findings were significantly associated with APS (On-line Table 3). The incidence of large territorial infarctions in the cerebellum was significantly higher in patients with APS than in those without APS $(P=.02)$. Localized cortical infarctions in the MCA area were significantly associated with APS $(P<.01)$. Bilateral but not unilateral borderzone infarcts were associated with APS $(P<.01)$. All basal ganglia lesions were in the anterior zone (7/7). Stenotic arterial lesions were seen in the ICA and MCA. WHM, lacunar infarcts, and the total number of patients with abnormal findings were significantly associated with age. Although acute microembolism in the cortex and/or subcortical white matter was associated with age $(P=.03)$, the association was not significant because the number of patients was small. We found that abnormal findings and lacunar infarcts were associated with patient age and APS status.

\section{DISCUSSION}

We compared brain MR findings in a large number of patients with SLE on the basis of their APS status. Our logistic regression analysis demonstrated that the incidence of large territorial infarcts, lacunar infarcts in the deep white matter, localized cortical infarcts in the MCA area, bilateral borderzone infarcts, anterior basal ganglia lesions, and stenotic arterial lesions was significantly higher in patients with SLE with APS than in those without APS, regardless of their age.

\section{Large Territorial Infarcts}

Arterial thrombosis rather than arterial sclerosis has been proposed as a major cause of territorial infarction in patients with APS. ${ }^{4,23}$ Our patients with and without APS manifested infarcts/infarct-like lesions in the absence of stenotic lesions in the cerebral arteries. This suggests thromboembolism as the main mechanism leading to lesions associated with cerebrovascular disease in patients with SLE with and without APS, though the presence of APS may further promote their development.

\section{Localized Cortical Infarcts}

The small arterial anastomoses between gyri of the cerebral cortex may help to prevent the development of cortical infarction. ${ }^{24}$ In patients with SLE, thromboembolism or small-vessel thrombosis promoted by the association of APS may result in the occlusion of small cortical arteries and the eventual development of localized infarcts.

\section{Bilateral Borderzone Infarcts}

Borderzone infarcts occur at characteristic locations at the junction between the external (cortical) and internal (subcortical) territory. Internal borderzone infarcts are thought to be attributable to hemodynamic compromise, ${ }^{25}$ whereas external borderzone infarcts have been attributed to embolism not necessarily associated with hypoperfusion. ${ }^{26,27}$ We observed both internal and external borderzone infarcts in patients with SLE without stenotic lesions on the relevant artery. Therefore, besides thromboembolism, different etiologies such as small-vessel injury may play a role in the development of borderzone infarcts. According to Atsumi et $\mathrm{al},{ }^{28}$ the induction of endothelin- 1 by aPL may contribute to an increase in the arterial tone and ultimately lead to vasospasm and the occlusion of small vessels. In 1 of our patients with borderzone infarction who presented with marked narrowing of the bilateral internal carotid arteries and the M1 segment of the bilateral MCA, vasculitis or arterial vasospasm was suspected. This suggests that hemodynamic compromise secondary to vasculitis or arterial vasospasm may represent a possible mechanism resulting in borderzone infarctions.

\section{Anterior Basal Ganglia Lesions}

Significantly more patients with SLE with APS than those without APS presented with anterior basal ganglia lesions. None of the patients with such lesions manifested stenotic lesions on the relevant MCA, suggesting that APS induces substantial neuronal injury caused by small-vessel injury. A clot of the M1 segment of the MCA often leads to caudate and/or putaminal 
infarction. Therefore, another explanation for basal ganglia lesions might be the infarction caused by the clot of the M1 segment after recanalization. Other factors, for example, demyelination, ${ }^{29}$ metabolic abnormalities, ${ }^{30}$ and vasculitis or vasospasm, ${ }^{28}$ must be considered as etiologic factors in the development of basal ganglia lesions. However, the mechanism(s) responsible for the high incidence of lesions in the anterior part remains to be identified.

\section{Stenotic Arterial Lesions}

We found that significantly more patients with SLE with APS than those without APS presented with stenotic arterial lesions, and these lesions were not associated with age. Moreover, in 1 of our patients, follow-up MRA study revealed resolution of the arterial stenosis. This suggests that the main cause of arterial stenosis is not associated with arteriosclerosis but with aPL/APS-related vasculitis or arterial vasospasm.

\section{White Matter Hyperintensity}

Compared with healthy individuals, WMH was a more common finding in patients with SLE and those with primary APS. ${ }^{31} \mathrm{Al}-$ though the specific mechanism, SLE per se or APS, resulting in the manifestation of WMH remains unknown, various pathologic conditions, not only small-vessel occlusion but also demyelination, may play a contributory role. The incidence of WMH was higher in our patients with SLE than in asymptomatic volunteers studied by others, ${ }^{29}$ but there was no significant difference among our patients with SLE with APS and those without APS. Moreover, WMH was significantly associated with age. The WMHs seen in patients with SLE are therefore not necessarily related to thromboembolism or small-vessel injury promoted by the presence of aPL.

\section{Miscellaneous Lesions}

The reported incidence of lacunar infarction in patients with aPL is $9 \% .{ }^{32}$ Our observation that this rate was higher in patients with SLE with APS than in those without APS (20\% versus 7\%) suggests that thromboembolism or small-vessel injury promoted by the presence of aPL may produce lacunar infarcts in patients with SLE with APS. Because lacunar infarcts were significantly associated with patient age and APS, however, they are not a characteristic finding in patients with SLE with APS. Whereas up to $42 \%$ of patients with SLE with uremia, thrombocytopenia, and hypertension have cerebral hemorrhages, ${ }^{33,34}$ their incidence in our patients with SLE with APS and those without APS was not different.

The presence of multiple microemboli implies that they arose at the same time, suggesting that their source is a proximal rather than a more distal independent microvascular occlusive event. Because the number of patients with cerebral hemorrhage and acute microemboli in the cortex and/or subcortical white matter was small in our study, we cannot comment on the difference in their incidence rate in patients with SLE with APS and those without APS.

Our retrospective study has some limitations. First, we evaluated patients with different phases of the disease as a single group, and we did not differentiate between acute and chronic infarctions. Moreover, we could not take neurologic symptoms into consideration because in most patients it was not possible to evaluate the association between their symptoms and MR findings.
Second, because many patients received therapy with antiplatelet, antihypertensive, and antilipemic drugs before undergoing MR imaging, we cannot rule out the possibility that these treatments affected our findings. Nonetheless, we posit that drug treatments did not play a major role because the clinical characteristics were not significantly different between patients with SLE with APS and those without APS. Third, because our study included neither healthy age-matched control subjects nor patients with primary APS, we did not assess the difference in the incidence of cerebral lesions in patients with SLE and control subjects. Fourth, we could not evaluate hemorrhagic lesions exactly because few of our patients underwent GRE T2WI. Last, we did not determine the incidence of large territorial infarcts, lacunar infarcts in the deep white matter, localized cortical infarcts in the territory of the MCA, bilateral borderzone infarcts, anterior basal ganglia lesions, and stenotic arterial lesions in patients with primary APS.

\section{CONCLUSIONS}

Our study of a large series of patients with SLE shows that APS is a significant risk factor for cerebral lesions. Our characterization of the spectrum of MR findings suggests that large territorial infarcts, lacunar infarcts in the deep white matter, localized cortical infarcts in the territory of the MCA, bilateral borderzone infarcts, anterior basal ganglia lesions, and stenotic arterial lesions are particularly common MR findings in patients with SLE with APS.

Disclosures: Kazuo Awai-UNRELATED: Consultancy: Daiichi-Sankyo Ltd; Employment: Toshiba Medical Systems, * Bayer Pharmaceutical, * Daiichi-Sankyo Ltd, * Eizai Ltd$^{*}$ ( ${ }^{*}$ money paid to institution).

\section{REFERENCES}

1. Johnson RT, Richardson EP. The neurological manifestations of systemic lupus erythematosus. Medicine 1968;47:337-69

2. O'Connor JF, Musher DM. Central nervous system involvement in systemic lupus erythematosus: a study of $\mathbf{1 5 0}$ cases. Arch Neurol 1966;14:157-64

3. Sibbitt WL Jr, Brooks WM, Kornfeld M, et al. Magnetic resonance imaging and brain histopathology in neuropsychiatric systemic lupus erythematosus. Semin Arthritis Rheum 2010;40:32-52

4. Kim JH, Choi CG, Choi SJ, et al. Primary antiphospholipid antibody syndrome: neuroradiologic findings in 11 patients. Korean J Radiol 2000;1:5-10

5. Golstein M, Meyer O, Bourgeois P, et al. Neurological manifestations of systemic lupus erythematosus: role of antiphospholipid antibodies. Clin Exp Rheumatol 1993;11:373-79

6. Bertolaccini ML, Gomez S, Pareja JF, et al. Antiphospholipid antibody tests: spreading the net. Ann Rheum Dis 2005;64:1639-43

7. Rodriguez Garcia JL, Khamashta MA. Clinical advances of interest in the diagnosis and treatment of patients with antiphospholipid syndrome. Rev Clin Esp 2013;213:108-13

8. Bertsias GK, Ioannidis JP, Aringer M, et al. Eular recommendations for the management of systemic lupus erythematosus with neuropsychiatric manifestations: report of a task force of the Eular Standing Committee for Clinical Affairs. Ann Rheum Dis 2010;69:2074-82

9. Harris EN, Gharavi AE, Asherson RA, et al. Cerebral infarction in systemic lupus: association with anticardiolipin antibodies. Clin Exp Rheumatol 1984;2:47-51

10. Harris EN, Gharavi AE, Mackworth, et al. Lupoid sclerosis: a possible pathogenetic role for antiphospholipid antibodies. Ann Rheum Dis 1985;44:281-83

11. Valdes-Ferrer SI, Vega F, Cantu-Brito C, et al. Cerebral changes in 
SLE with or without antiphospholipid syndrome: a case-control MRI study. J Neuroimaging 2008;18:62-65

12. Hochberg MC. Updating the American College of Rheumatology revised criteria for the classification of systemic lupus erythematosus. Arthritis Rheum 1997;40:1725

13. Wilson WA, Gharavi AE, Koike T, et al. International consensus statement on preliminary classification criteria for definite antiphospholipid syndrome: report of an international workshop. Arthritis Rheum 1999;42:1309-11

14. Lodder J, Hupperts R, Boreas A, et al. The size of territorial brain infarction on CT relates to the degree of internal carotid artery obstruction. J Neurol 1996;243:345-49

15. Bang OY, Ovbiagele B, Liebeskind DS, et al. Clinical determinants of infarct pattern subtypes in large vessel atherosclerotic stroke. J Neurol 2009;256:591-99

16. Kimura K, Minematsu K, Koga M, et al. Microembolic signals and diffusion-weighted MR imaging abnormalities in acute ischemic stroke. AJNR Am J Neuroradiol 2001;22:1037-42

17. Bogousslavsky J, Regli F. Unilateral watershed cerebral infarcts. Neurology 1986;36:373-77

18. Damasio H. A computed tomographic guide to the identification of cerebral vascular territories. Arch Neurol 1983;40:138-42

19. Fisher CM. Lacunar strokes and infarcts: a review. Neurology 1982;32:871-76

20. Fazekas F, Kleinert R, Offenbacher $\mathrm{H}$, et al. The morphologic correlate of incidental punctate white matter hyperintensities on MR images. AJNR Am J Neuroradiol 1991;12:915-21

21. Wuerfel J, Haertle M, Waiczies $\mathrm{H}$, et al. Perivascular spaces: MRI marker of inflammatory activity in the brain? Brain 2008;131:2332-40

22. Chao CP, Kotsenas AL, Broderick DF. Cerebral amyloid angiopathy: CT and MR imaging findings. Radiographics 2006;26:1517-31

23. Aron AL, Gharavi AE, Shoenfeld Y. Mechanisms of action of antiphospholipid antibodies in the antiphospholipid syndrome. Int Arch Allergy Immunol 1995;106:8-12
24. Duvernoy HM, Delon S, Vannson JL. Cortical blood vessels of the human brain. Brain Res Bull 1981;7:519-79

25. Derdeyn CP, Khosla A, Videen TO, et al. Severe hemodynamic impairment and border zone-region infarction. Radiology 2001;220: 195-201

26. Torvik A. The pathogenesis of watershed infarcts in the brain. Stroke 1984;15:221-23

27. Chaves CJ, Silver B, Schlaug G, et al. Diffusion- and perfusionweighted MRI patterns in borderzone infarcts. Stroke 2000; 31:1090-96

28. Atsumi T, Khamashta MA, Haworth RS, et al. Arterial disease and thrombosis in the antiphospholipid syndrome: a pathogenic role for endothelin 1. Arthritis Rheum 1998;41:800-07

29. Karussis D, Leker RR, Ashkenazi A, et al. A subgroup of multiple sclerosis patients with anticardiolipin antibodies and unusual clinical manifestations: do they represent a new nosological entity? Ann Neurol 1998;44:629-34

30. Sabet A, Sibbitt WL Jr, Stidley CA, et al. Neurometabolite markers of cerebral injury in the antiphospholipid antibody syndrome of systemic lupus erythematosus. Stroke 1998;29:2254-60

31. Hachulla E, Michon-Pasturel U, Leys D, et al. Cerebral magnetic resonance imaging in patients with or without antiphospholipid antibodies. Lupus 1998;7:124-31

32. Provenzale JM, Barboriak DP, Allen NB, et al. Patients with antiphospholipid antibodies: $\mathrm{CT}$ and $\mathrm{MR}$ findings of the brain. $A J R$ 1996;167:1573-78

33. Asherson RA, Mercey D, Phillips G, et al. Recurrent stroke and multi-infarct dementia in systemic lupus erythematosus: association with antiphospholipid antibodies. Ann Rheum Dis 1987; 46:605-11

34. Provenzale JM, Heinz ER, Ortel TL, et al. Antiphospholipid antibodies in patients without systemic lupus erythematosus: neuroradiologic findings. Radiology 1994;192:531-37 